\title{
Efficacy and safety of human papillomavirus vaccine for primary prevention of cervical cancer: A review of evidence from phase III trials and national programs
}

\author{
Partha Basu, Dipanwita Banerjee, Priyanka Singh, Chandrani Bhattacharya, Jaydip Biswas'
}

\begin{abstract}
The Human Papillomavirus (HPV) vaccines have been widely introduced in the national immunization programs in most of the medium and high income countries following endorsement from national and international advisory bodies. HPV vaccine is unique and its introduction is challenging in many ways - it is the first vaccine developed to prevent any cancer, the vaccine is gender specific, it targets adolescent females who are difficult to reach by any health intervention programs. It is not unusual for such a vaccine to face scepticism and reservations not only from lay public but also from professionals in spite of the clinical trial results convincingly and consistently proving their efficacy and safety. Over the last few years millions of doses of the HPV vaccine have been administered round the world and the efficacy and safety data have started coming from the real life programs. A comprehensive cervical cancer control program involving HPV vaccination of the adolescent girls and screening of the adult women has been proved to be the most cost-effective approach to reduce the burden of cervical cancer. The present article discusses the justification of HPV vaccination in the backdrop of natural history of cervical cancer, the mechanism of action of the vaccines, efficacy and safety data from phase III randomized controlled trials as well as from the national immunization programs of various countries.
\end{abstract}

Key words: Human Papillomavirus vaccine, efficacy, safety, Phase III randomized trials, national immunization programs

\section{Introduction}

The International Agency for Research on Cancer (Lyon, France) recognized Human Papillomavirus (HPV) as the 'necessary' cause of cervical cancer and HPV types 16 and 18 as carcinogenic agents for humans based on the strong and consistent associations between the infections and the disease. ${ }^{[1]}$ The recognition of the etiologic role of carcinogenic HPV types paved the way for a novel primary prevention strategy against cervical cancer through vaccines targeting the virus. India ranking number one globally in terms of cervical cancer burden has an estimated 134,420 new cases of cervical cancer every year and 72,825 deaths from the disease. ${ }^{[2]}$ In spite of some reduction in incidence in recent years in the urban population, possibly owing to raised average age at marriage, reduction in number of child births and high number of hysterectomies, the fact remains that the disease is the leading cancer killer of Indian women of reproductive age. A comprehensive approach to cervical cancer prevention and control involving health education,

Departments of Gynaecologic Oncology and 'Surgical Oncology, Chittaranjan National Cancer Institute, Kolkata, West Bengal, India

Correspondence to: Dr. Partha Basu,

E-mail: basupartha@hotmail.com

\begin{tabular}{|l|l|}
\hline \multicolumn{2}{|c|}{ Access this article online } \\
\hline Quick Response Code: & Website: \\
\hline & www.sajc.org \\
\cline { 2 - 2 } & \\
\hline
\end{tabular}

vaccinating girls before initiation of sexual activity, screening women for precancerous lesions and treatment before progression to invasive disease can significantly reduce the morbidity and mortality from the disease.

\section{HPV in Natural History of Cervical Carcinogenesis}

HPV is the most common sexually transmitted infection (STI). Women are at highest risk of acquiring HPV when they initiate their sexual life. Peak prevalence of infection is observed between 20 and 25 years and the prevalence comes down drastically after 30 years of age since most of the infected women clear the infection due to natural immunity. More than 90 percent of the immune-competent women have been found to clear the HPV 16 infection within 5 years without any treatment. ${ }^{[3]}$ Women who cannot clear the infection and have persistently infected cervix are at the highest risk of developing cervical cancer.

The papillomavirus infects the basal keratinocytes through micro-abrasions present in the cervical epithelium. If there is persistent infection, the viral genome gets integrated to the host genome that over-expresses two onco-proteins, E6 and E7. The E6 proteins degrade p53 genes resulting in genetic instability and accumulation of mutated deoxyribonucleic acid (DNA) that trigger uncontrolled cellular multiplication. ${ }^{[4]}$ The E7 protein degrades the active form of retinoblastoma protein leading to the progression of the cell into the S-phase of the cell cycle with subsequent unregulated cell replication. ${ }^{[5]}$ Thus unregulated cellular proliferation in the affected epithelium leads to development of cervical neoplasia.

Cervical cancer is preceded by HPV induced premalignant condition known as cervical intraepithelial neoplasia (CIN), 
graded from CIN 1 to CIN 3 depending on the severity. While majority of CIN 1 lesions are self-limiting, CIN 2 and CIN 3 are considered true pre-malignant lesions. Given adequate follow up time, specially the CIN3 lesions, 30 percent to 50 percent of them will progress to invasive cancers. ${ }^{[6]}$ Cervical cancer screening detects the disease at the CIN 2/3 stage when appropriate interventions prevent further progression to invasive cervical cancer (ICC). The vaccines prevent even the development of CIN $2 / 3$ by preventing persistent infection of certain high risk HPVs, thus eliminating the chance of development of invasive cancer in the future by those HPV types.

\section{Mechanism of Action of the HPV Vaccines}

The HPV vaccines currently available are - the bivalent vaccine (Cervarix ${ }^{\mathrm{TM}}$, GlaxoSmithKline Biologicals) and the quadrivalent vaccine (Gardasil ${ }^{\mathrm{TM}}$, Merck). These vaccines aim to prevent infection from HPV types 16 and 18 , since these two types are most carcinogenic and are responsible for majority of cervical cancers. The risk of developing squamous cell carcinoma of cervix is 435 times higher if someone is infected by HPV 16 and 248 times higher if someone is infected by HPV 18, compared to a non-infected individuals. ${ }^{[7]}$ The proportion of cervical cancers attributed to HPV 16/18 ranges from 66.8 percent in sub-saharan Africa to 84.3 percent in Europe and America ${ }^{[8]}$ In an Indian study encompassing four different zones, type 16 alone was detected in 57.5 percent of cervical cancers, type 18 alone in 10.4 percent, and both types were detected in additional 7.3 percent. ${ }^{[9]}$ The fact that nearly 70 percent of all cervical cancers in India are attributed to HPV 16/18 signifies that, if the vaccines are 100 percent effective in preventing HPV 16/18 infections and if administered prior to exposure to HPV infections, the maximum quantum of protection that can be expected is around 70 percent. The vaccinated girls are still at risk of developing cervical cancer from the HPV types other than types $16 / 18$, though such risk is much lower.

The antigens for both the vaccines are virus like particles (VLP) derived from the L1 surface protein of the respective types of the virus. The VLPs are non-pathogenic and cannot infect cells, since they do not have viral genome. The vaccines induce high titer of serum immunoglobulin $\mathrm{G}$ antibody against respective HPV types, which is secreted in the cervico-vaginal secretion and is also exuded from the micro-abrasions in the epithelium. Presence of the antibodies at the point of viral entry ensures the neutralization of the virus before it gets an opportunity to bind to infect the basal keratinocytes. ${ }^{[10]}$

\section{Efficacy of the HPV Vaccines in Phase III trials}

Both bivalent and the quadrivalent vaccines have been rigorously evaluated through phase III randomized placebo controlled trials (RCT). The primary efficacy end point for these studies was prevention of CIN 2 or worse disease. An advisory committee of the World Health Organization (WHO) (in which the pharmaceutical agencies had no role to play) agreed upon such endpoint as surrogate for cervical cancer, since it is not ethically acceptable to allow any subject of a study to be followed up until she develops ICC. ${ }^{[1]}$ The secondary efficacy end point was prevention of type-specific persistent infection, which is an obligate precursor of cervical neoplasia.

Till date two phase III RCTs have evaluated the bivalent vaccine and three phase III RCTs have evaluated the quadrivalent vaccine. ${ }^{[12]}$ In the phase III trials there were subjects without any evidence of HPV infection or cytological abnormality and also subjects with active or past HPV infection and even cytological abnormalities. To compensate for the expected difference in outcomes in these two major subgroups, the trials analyzed the vaccine efficacy in two different efficacy cohorts - per-protocol population and the intention-to-treat population. The per-protocol population included those who neither had positive serology (indicative of past infection) nor DNA in cervix (indicates active infection) for the target HPV types and received three doses of the vaccines within one year. This population in real life represents young sexually naïve subjects receiving full doses of the vaccines. The Intention to treat analysis included subjects who might or might not have evidence of past or active HPV infection and received at least one dose of the vaccine. Some of them even had abnormal cytology at the time of entry in the studies. This cohort represents a mixed population of sexually naïve and sexually active girls or women who may or may not receive complete dose of the vaccines. The efficacies of the vaccines observed in the RCTs should be judged in the light of these two efficacy cohorts reflecting two different real life scenarios. The summary of the efficacies of the vaccines for various endpoints from the phase III trials is given in Table 1.

As per the WHO guidelines the primary target for the HPV vaccine for the national immunization programs should be girls aged between 9 years and 13 years. ${ }^{[13]}$ The efficacy of the vaccines will be similar to the per-protocol analysis population rather than the intention-to-treat population in this sexually naïve population if they receive three doses of the vaccine. There is an exciting possibility that even two doses of the vaccine may be as protective as three doses, going by the initial results of the Costa Rican phase III trial. ${ }^{[14]}$

Concerns have been expressed about the possible increase in the risk of cervical neoplasias in the women with HPV infection (past or present) from quadrivalent vaccination. ${ }^{[15]}$ As discussed earlier, in the phase III quadrivalent vaccine trials at the study entry nearly 1 percent women in vaccine and placebo group had high grade squamous intra-epithelial lesions (HSIL) or atypical squamous cells - cannot exclude high grade squamous intra-epithelial lesions (ASC-H) on cytology and more than 5 percent 
Table 1: Vaccine efficacy against various end-points in per-protocol and intention to treat cohorts from phase III RCTs-Risk ratio of zero indicates 100 percent efficacy, whereas one indicates no difference with controls ${ }^{[12]}$

\begin{tabular}{|c|c|c|c|c|c|}
\hline \multirow[t]{2}{*}{ Efficacy endpoint } & \multicolumn{2}{|c|}{ Vaccine } & \multicolumn{2}{|c|}{ Control } & \multirow{2}{*}{$\begin{array}{c}\text { Risk ratio } \\
\text { (95 percent } \text { C.I.) }\end{array}$} \\
\hline & No. of subjects & No. of events & No. of subjects & No. of events & \\
\hline \multicolumn{6}{|l|}{ Persistent HPV 16 infection } \\
\hline Per-protocol population & 7332 & 31 & 7153 & 475 & $0.06[0.04-0.09]$ \\
\hline Intention to treat population & 5974 & 25 & 5990 & 173 & $0.15[0.10-0.23]$ \\
\hline \multicolumn{6}{|l|}{ Persistent HPV 18 infection } \\
\hline Per-protocol population & 7056 & 9 & 6952 & 193 & $0.05[0.03-0.09]$ \\
\hline Intention to treat population & 6456 & 16 & 6492 & 69 & $0.24[0.14-0.42]$ \\
\hline \multicolumn{6}{|l|}{ HPV 16 associated CIN 2+ } \\
\hline Per-protocol population & 11617 & 3 & 11323 & 93 & $0.04[0.01-0.11]$ \\
\hline Intention to treat population & 14506 & 85 & 14523 & 232 & $0.47[0.36-0.61]$ \\
\hline \multicolumn{6}{|l|}{ HPV 18 associated CIN 2+ } \\
\hline Per-protocol population & 11849 & 2 & 11716 & 26 & $0.10[0.03-0.38]$ \\
\hline Intention to treat population & 14023 & 8 & 14030 & 53 & $0.16[0.08-0.34]$ \\
\hline \multicolumn{6}{|c|}{ CIN2+ associated with HPV 31/33/45/52/58 } \\
\hline Per-protocol population & 12478 & 74 & 12533 & 130 & $0.58[0.43-0.77]$ \\
\hline Intention to treat population & 17213 & 267 & 17263 & 341 & $0.79[0.72-0.83]$ \\
\hline
\end{tabular}

$\mathrm{CIN}=$ Cervical intraepithelial neoplasia, HPV=Human papillomavirus, RCTs=Randomized placebo controlled trials

had low grade squamous intra-epithelial lesions (LSIL) on cytology. They were included in the intention-to-treat analysis and obviously some of them in either group went on to develop CIN 2 or CIN 3 lesions. The vaccinated group did have higher incidence of CIN 2/3 (though the difference was not significant as evident by wide confidence intervals) compared with the placebo group. The baseline demographic analysis showed that the vaccinated group had higher prevalence of other risk factors like current smokers, other STIs and most importantly HSIL cytology at baseline (6.5 percent vs 3.7 percent). This imbalance in baseline demographic characteristics and the small number of events was responsible for the observed discrepancy in the sub-group analysis. In subsequent studies of quadrivalent vaccines and all studies with bivalent vaccines where the subgroups were more balanced in terms of the other risk factors, no such increase in risk of neoplasias was observed. All these information was presented to Food and Drug Administration (FDA) prior to obtaining the license and are still available in the public domain. ${ }^{[16]}$

\section{Efficacy of HPV Vaccine in National Immunization Programs}

WHO recommended in 2007 that HPV vaccination of 9-13 year old girls should be considered as part of comprehensive cervical cancer control through effective, affordable and equitable delivery strategies. ${ }^{[17]}$ Until February 2013 the vaccine is part of the National Immunization Programs of 55 countries that include almost all countries in North America and Europe, Australia, New Zealand, Argentina, Chile, Mexico and Peru in Latin America, Singapore, Bhutan, Japan and Malaysia in Asia. ${ }^{[18]}$ Actual population impact of HPV vaccination on the incidence of HPV-related diseases is currently available from many of these country programs.
Australia was the first country in the world to provide free quadrivalent HPV vaccines (protecting against genital warts as well as cervical cancer) to 12 to 18 year old girls starting from mid-2007. The earliest effect of the HPV vaccination is evident from the protection from genital warts since the natural history of the warts is much shorter compared to cervical neoplasias. The very first report of reduction of incidence of genital wart in the vaccinated population was from the sexual health clinics in Melbourne, Australia. ${ }^{[19]}$ In the vaccinated cohort of women below 21 years there was drastic reduction in the incidence of genital warts compared to the older non-vaccinated cohort. Data from the Victoria Cervical Cytology Registry, Australia showed that within 3 years after the introduction of the vaccination program, the incidence of high grade abnormalities in young women became nearly half of that reported before the introduction of the vaccine. ${ }^{[20]}$ The first effectiveness study of the quadrivalent vaccine from an entire country was reported from Sweden in 2013. [21] The efficacy against genital warts in the subjects vaccinated before the age of 14 years (93 percent) in Sweden was in fact higher than the efficacy among HPV-naive subjects reported in the quadrivalent HPV vaccine clinical trials (83 percent).

The most robust evidence of the effectiveness of the vaccine in cervical cancer prevention was obtained from Finland. ${ }^{[22]}$ The participants of the FUTURE II and FUTURE III studies (to evaluate the quadrivalent vaccines) from Finland were followed up after completion of the trials in 2007 through the well-organized Finnish cancer Registry over the next five years. The study participants were unblinded, and the placebo recipients were offered the quadrivalent vaccine. Nearly half of the placebo recipients were vaccinated at this stage. An age-matched reference unvaccinated cohort was linked with the cancer registry as the control group to identify all cases of CIN3 and ICC 
during the follow up. During the follow up no CIN3 or cervical cancer was detected in the vaccinated cohort, three CIN3 cases but no cancers were detected in the original placebo cohort (nearly 50 percent were vaccinated). In the unvaccinated reference cohort $59 \mathrm{CIN} 3$ and three cervical cancers were detected. CIN3+ incidence rates of $0 / 100,000$ person years in the original vaccinated cohort was in stark contrast to the incidence rate of $93.8 / 100,000$ person years in the unvaccinated reference cohorts, thus proving the long term (nearly 10 years) protection offered by the HPV vaccine against the most valid end point of CIN3 and invasive cancers.

\section{Safety Data from Phase III trials}

All RCTs reported serious and non-serious adverse events (AE) in similar fashions following standard good clinical practice guidelines from more than 20,000 trial participants. Pain at the injection site, headache and fatigue were the most frequently reported vaccine related AEs. The serious adverse events (SAE) were reported in similar frequency in both the vaccine and control arms (pooled relative Risk for participants having $\mathrm{SAE}=1.00 ; 95$ percent CI 0.91-1.09) suggesting no additional risk of SAE due to HPV vaccination. ${ }^{[12]}$ SAEs that were considered to be vaccine related were rare.

For any new vaccine there are concerns about vaccine-induced medical conditions, especially systemic autoimmune disorders and neurological disorders. The long term follow up of the subjects participating in the RCTs did not have higher incidence of these conditions compared with the control group. ${ }^{[23]}$ The Center for Disease Control (CDC), Atlanta, USA observed that the reported number of cases of Guillain-Barré syndrome was within the range expected by chance alone in the population. ${ }^{[2]}$ Of the few reported deaths in the studies, causes of death were consistent with causes expected in the general adolescent and adult populations, and were not judged to be vaccine-related, not only by the investigators but also by the Global Advisory Committee on Vaccine Safety (GACVS) of WHO. ${ }^{[25]}$

\section{Safety Data from National Immunization Programs}

In Australia millions of doses of the vaccine have been administered and the most serious adverse event reported is anaphylactic and allergic reactions. The current estimated rate of anaphylaxis based on doses given in Australia is 1.7 cases per million doses. ${ }^{[26]}$ This rate is comparable to the rates for anaphylaxis for other vaccines given to children and adolescents, which range from 0 to 3.5 per million doses. ${ }^{[25]}$

As of September, 2011, approximately 40 million doses of Quadrivalent vaccine were distributed in the USA and Vaccine Adverse Event Reporting System (VAERS) in the country received a total of 20,096 reports of adverse events of which 8 percent were considered serious. ${ }^{[27]}$ Thorough analysis of the all these reports by regulatory bodies like FDA and CDC did not observe any major or unexpected adverse events that could be ascribed to the vaccine nor did they find any consistent pattern of such events to suggest a causal association. These included the reported cases of Guillain-Barré syndrome, thrombo-embolism, and deaths.

In the United Kingdom, the Commission on Human Medicines (CHM) and the Medicines and Healthcare Products Regulatory Agency (MHRA) reviewed the safety of Bivalent vaccine two years after its introduction in the national program. They concluded that no serious new risks have been identified during its extensive use over 2 years. ${ }^{[28]}$

A typical instance of the high alertness of the regulatory bodies was evident recently, when CDC and FDA convened a Clinical Immunization Safety Assessment (CISA) working group to investigate the deaths owing to suspected cerebral vasculitis following vaccination in USA reported in 2012. ${ }^{[29]}$ After a thorough review and discussion of the article published in a not so highly rated journal, the working group identified substantial methodological concerns and lack of evidence to support the authors' conclusions that the patients had vasculitis due to vaccination, or that HPV vaccine was causally associated with deaths from cerebral vasculitis. ${ }^{[30]}$ We need to understand that deaths due to various causes including accidents do occur even in apparently healthy young females. To ascribe the deaths to any intervention (HPV vaccination in this case) certain conditions need to be fulfilled - proportion of deaths should be higher in the intervention group than that observed in non-intervention group, there should be a consistent pattern and a biologically plausible explanation after ruling out other more apparent causes. Following these criteria none of the deaths initially suspected due to vaccination (deaths due to accidents, snakebites or suicides in India, due to the rupture of an intra-thoracic tumor in UK etc.) could be linked to the vaccines.

\section{Cost Effectiveness of HPV Vaccines}

The cost-effectiveness study of all possible options for cervical cancer prevention showed that the most effective strategy with the lowest incremental cost-effectiveness ratio per quality adjusted life year is one combining HPV vaccination at age 12 years with triennial conventional cytologic screening beginning at age 25 years. ${ }^{[31]}$ Cost-effectiveness should be looked at in the context of the negotiated price of the vaccine specific for the country, subsidies obtained from international agencies, financial cost of the delivery strategy vis-à-vis the treatment cost of advanced cancer, social and financial loss owing to losing mothers and wives at their prime and cost of disabilities resulting from advanced cancer. The prices at which the vaccines are sold in the private market are being drastically reduced for national programs. Hard bargaining with the manufactures can possibly reduce the cost of per dose for a huge market as in India to less the 
$1 \$$, a price that is considered affordable for immunization program in the country. India can seek support from Global Alliance for Vaccines and Immunization (GAVI Alliance) since the Alliance has already committed to donate the vaccines to eligible countries. Other important vaccines, e.g., hepatitis B vaccine, have been introduced in National Immunization Program in India with donations from GAVI and subsequently could be sustained due to the drastic price reduction through indigenous productions.

\section{Conclusions}

WHO, United Nations Populations Fund (UNFPA), International Union Against Cancer (UICC), International Federation of Gynecologists and Obstetricians (FIGO) and other organizations that influence public health policies globally have unanimously endorsed HPV vaccines as an effective cancer prevention option. The Strategic Advisory Group of Experts (SAGE) on immunization, which reports to the Director-General of WHO on issues ranging from vaccine research and development to immunization delivery, surmised that the introduction of vaccines is likely to bring great benefits worldwide, particularly to those developing countries where cervical cancer is a major cause of death, and screening programs are limited or absent. ${ }^{[32]}$ It is time that the health policy makers in the countries with high cervical cancer burden like India, make an informed decision and seriously consider the introduction of both HPV vaccination and organized screening as part of comprehensive cervical cancer control program.

\section{References}

1. Bosch FX, Lorincz A, Muñoz N, Meijer N, Shah KV. The causal relation between human papillomavirus and cervical cancer. J Clin Pathol 2002; 55;244-65.

2. Available from: http://apps.who.int/hpvcentre/statistics/ dynamic/ico/country_pdf/IND.pdf. Updated in September 2010. [Last accessed on 2013 May 13].

3. Richardson H, Abrahamowicz M, Tellier PP, Kelsall G, du Berger R, Ferenczy A, et al. Modifiable risk factors associated with clearance of type-specific cervical human papillomavirus infections in a cohort of university students. Cancer Epidemiol Biomarkers Prev 2005; 14:1149-56.

4. Mantovani F, Banks L. The human papillomavirus E6 protein and its contribution to malignant progression. Oncogene 2001;20:7874-87.

5. Boyer SN, Wazer DE, Band V. E7 protein of human papilloma virus-16 induces degradation of retinoblastoma protein through the ubiquitin-proteasome pathway. Cancer Research 1996;56:4620-4.

6. McCredie MR, Sharples KJ, Paul C, Baranyai J, Medley G, Jones RW, et al. Natural history of cervical neoplasia and risk of invasive cancer in women with cervical intraepithelial neoplasia 3: A retrospective cohort study. Lancet Oncol 2008;9:425-34.

7. Muñoz N, Bosch FX, de Sanjosé S, Herrero R, CastellsaguéX, Shah KV, et al. Epidemiologic classification of human papillomavirus types associated with cervical cancer. N Engl J Med 2003;348:518-27.

8. Muñoz N, Bosch FX, Castellsagué X, Díaz M, de Sanjose S, Hammouda D, et al. Against which human papillomavirus types shall we vaccinate and screen? The international perspective. Int J Cancer 2004; 111:278-85.

9. Basu P, Chandna P, Bamezai RN, Siddiqi M, Saranath D, Lear A, et al. MassARRAY spectrometry is more sensitive than PreTect HPV-Proofer and consensus PCR for type-specific detection of high-risk oncogenic human papillomavirus genotypes in cervical cancer. J Clin Microbiol 2011;49:3537-44.

10. Stanley M. Immune responses to human papillomavirus. Vaccine 2006;24(Suppl 1):S16-22.

11. Pagliusi SR, Aguado MT. Efficacy and other milestones for human papillomavirus vaccine introduction. Vaccine 2004;23:569-78.

12. Lu B, Kumar A, Castellsagué X, Giuliano AR. Efficacy and Safety of Prophylactic Vaccines against Cervical HPV Infection and Diseases among Women: A Systematic Review and Meta-Analysis. BMC Infect Dis 2011;11: 13.

13. World Health Organization. WHO guidance note: Comprehensive cervical cancer prevention and control: A healthier future for girls and women. WHO Press, World Health Organization, Geneva, Switzerland, 2013.

14. Kreimer AR, Rodriguez AC, Hildesheim A, Herrero R, Porras C, Schiffman M, et al. Proof-of-Principle Evaluation of the Efficacy of Fewer Than Three Doses of a Bivalent HPV 16/18 Vaccine. J Natl Cancer Inst 2011; 103:1444-51.

15. Tomljenovic L, Shaw CA, Spinosa JP. Human Papillomavirus (HPV) Vaccines as an Option for Preventing Cervical Malignancies: (How) Effective and Safe? Current Pharmaceutical Design 2013;19:1-10.

16. Available from: http://www.fda.gov/ohrms/dockets/ac/06/ briefing/2006-4222B3.pdf. [Last accessed on 13 May 2013].

17. World Health Organization. Global Advisory Committee on Vaccine Safety, 2007. Wkly Epidemiol Rec 2007;3:17-24.

18. Available from: www.cervicalcanceraction.org/comments/ comments3.php. [Last accessed on 2013 Apr 12].

19. Read TR, Hocking JS, Chen MY, Donovan B, Bradshaw CS, Fairley CK. The near disappearance of genital warts in young women 4 years after commencing a national human papillomavirus (HPV) vaccination program. Sex Transm Infect 2011;87:544-7.

20. Brotherton JM, Fridman M, May CL, Chappell G, Saville AM, Gertig DM. Early effect of the HPV vaccination program on cervical abnormalities in Victoria, Australia: An ecological study. Lancet 2011;377:2085-92.

21. Leval A, Herweijer E, Ploner A, Eloranta S, Fridman Simard J, Dillner J, et al. Quadrivalent Human Papillomavirus Vaccine Effectiveness: A Swedish National Cohort Study. J Natl Cancer Inst 2013; 105:469-74.

22. Rana MM, Huhtala H, Apter D, Eriksson $T$, Luostarinen $T$, Natunen $\mathrm{K}$, et al. Understanding long-term protection of human papillomavirus vaccination against cervical carcinoma: Cancer registry-based follow-up. Int J Cancer 2013;132:2833-8.

23. Einstein MH, Baron M, Levin MJ, Chatterjee A, Fox B, Scholar S, et al. Comparative immunogenicity and safety of human papillomavirus (HPV)-16/18 vaccine and HPV-6/11/16/18 vaccine: Follow-up from months 12-24 in a Phase III randomized study of healthy women aged 18-45 years. Hum Vaccin 2011;7: 1343-58.

24. Centers for Disease Control and Prevention. Vaccine Safety - Human Papillomavirus (HPV) Vaccine. Available from: http://www.cdc.gov./vaccinesafety/Vaccines/HPV/Index. html. [Last accessed on 2013 May 13].

25. World Health Organization. Global Vaccine Safety. Observed rate of vaccine reactions, Human Papillomavirus Vaccine. Available from: http://www.WHO.int/nuvi/hpv/VaccRate_Infosheet_Poster. pdf. [Last accessed on 2013 May 13].

26. Gee J, Naleway A, Shui I, Baggs J, Yin R, Li R, et al. Monitoring the safety of quadrivalent human papillomavirus vaccine: Findings from the vaccine safety datalink. Vaccine 2011;29:8279-84.

27. Available from: http://www.cdc.gov/vaccinesafety/vaers/ gardasil.htm. [Last accessed on 2013 Apr 12].

28. MHRA Public Assessment Report. Cervarix (HPV vaccine): Update on UK safety covering the first two years of the HPV immunisation program, October 2010. Available from: http://www.mhra.gov. uk. [Last accessed on 2013 May 13].

29. Tomljenovic L, Shaw CA. Death after quadrivalent human papillomavirus (HPV) vaccination: Causal or coincidental? Pharmaceutical regulatory affairs. Open Access 2012;S12:001. Available from: http://dx.doi.org/10.4172/2167-7689. S12-001. [Last accessed on 2013 May 13].

30. Review of a Published Report of cerebral vasculitis after vaccination with the Human Papillomavirus (HPV) Vaccine. CDC Vaccine Safety. November 9, 2012. Available from: http:// 
www.cdc.gov/vaccinesafety/Activities/cisa/technical_report. html. Technical Report-Vaccine Safety. November 9, 2012. [Last accessed on 2013 May 13].

31. Goldie SJ, Kohli M, Grima D, Weinstein MC, Wright TC, Bosch FX, et al. Projected clinical benefits and cost-effectiveness of a human papillomavirus 16/18 vaccine. J Natl Cancer Inst 2004;96:604-15.

32. World Health Organization. Human papillomavirus vaccines WHO position paper. Wkly Epidemiolo Rec 2009;84:117-32.
How to cite this article: Basu P, Banerjee D, Singh P, Bhattacharya C, Biswas J. Efficacy and safety of human papillomavirus vaccine for primary prevention of cervical cancer: A review of evidence from phase III trials and national programs. South Asian J Cancer 2013;2: 187-92.

Source of Support: Nil. Conflict of Interest: Declaration of Dr. Partha Basu has been a member of the advisory boards of both GlaxoSmithKline and Merck. He has been principal investigators for two HPV vaccine trials sponsored by GlaxoSmithKline. 